Re-submitted to Physics of Fluids, 1 October 2011

\title{
Molecular dynamics study of the processes in the vicinity of the \\ n-dodecane vapour/liquid interface
}

Jian-Fei Xie, ${ }^{1}$ Sergei S. Sazhin,, ${ }^{,{ }^{*}}$ and Bing-Yang $\mathrm{Cao}^{2}$

${ }^{1}$ Sir Harry Ricardo Laboratories, School of Computing, Engineering and Mathematics, University of Brighton, Cockcroft Building, Brighton BN2 4GJ, UK

${ }^{2}$ Key Laboratory for Thermal Science and Power Engineering of the Ministry of Education, Department of Engineering Mechanics, Tsinghua University, Beijing 100084, China

* Corresponding author, e-mail: s.sazhin@brighton.ac.uk. 


\begin{abstract}
:
Molecular dynamics simulation is used to study the evaporation and condensation of n-dodecane $\left(\mathrm{C}_{12} \mathrm{H}_{26}\right)$, the closest approximation to Diesel fuel. The interactions in chain-like molecular structures are modelled using an optimised potential for liquid simulation (OPLS). The thickness of the transition layer between the liquid and vapour phases at equilibrium is estimated. It is shown that molecules at the liquid surface need to obtain relatively large translational energy to evaporate. The vapour molecules with large translational energy can easily penetrate deeply into the transition layer and condense in the liquid phase. The evaporation/condensation coefficient is estimated and the results are shown to be compatible with the previous estimates based on the molecular dynamics (MD) analysis and the estimate based on the transition state theory. The velocity distribution functions of molecules at the liquid-vapour equilibrium state are found in the liquid phase, interface and the vapour phase. These functions in the liquid phase and at the interface are shown to be close to isotropic Maxwellian for all velocity components. The velocity distribution function in the vapour phase is shown to be close to bi-Maxwellian with the temperature for the distribution normal to the interface being larger than the one for the distribution parallel to the interface.
\end{abstract}

Keywords: Molecular dynamics simulation, Evaporation, Condensation, Translational energy, Velocity distribution function, Kinetic modelling 


\section{INTRODUCTION}

An almost universal engineering approach to modelling the evaporation/condensation processes is based on the hydrodynamic approximation. In this approximation, it is assumed that vapour at the liquid/gas interface is always saturated, which allows one to reduce the modelling of the evaporation/condensation process to the modelling of vapour diffusion/convection from the droplet surface to ambient gas [1-8]. The limitation of this approach, even in the case of evaporation at high pressures has been discussed in our previous papers [9-13]. In these papers the evaporation of n-dodecane (the nearest approximation for Diesel fuel) has been considered and a new model for the analysis of droplet heating and evaporation has been developed based on the combination of the kinetic and hydrodynamic approaches. In the immediate vicinity of droplet surfaces (up to about one hundred molecular mean free paths), the vapour and ambient gas dynamics have been studied based on the Boltzmann equation (kinetic region), while at larger distances the analysis has been based on the hydrodynamic equations (hydrodynamic region). Mass, momentum and energy fluxes have been conserved at the interface between these regions. The predictions of this model have been shown to be as accurate as those of the model based on the kinetic equations in the whole domain, but both have differed considerably (up to 10\%) from the predictions of the hydrodynamic models for Diesel engine-like conditions. The practical application of this combined or kinetic modelling, however, requires the specification of boundary conditions at the droplet surface and at the interface between the kinetic and hydrodynamic regions. These boundary conditions include the evaporation/condensation coefficient at the droplet surface and the specification of the distribution functions of molecules leaving the droplet surface or entering the kinetic region from the hydrodynamic one. In the above mentioned papers [9-13], it has been implicitly or explicitly assumed that this distribution function is isotropic Maxwellian in both cases (cf. Eq. (15) of Ref. [13]). The values of the evaporation/condensation coefficients have been assumed equal to 0.04 and 0.5 (the minimal and average value of this parameter of water) in Ref. [9] or 1 in Refs. [10-13]. None of these assumptions could be rigorously justified. The only practical way to perform this justification is to base it on the molecular dynamic simulation at the interface region.

The interface between the gas and its condensed phase (liquid) is typically a couple of molecular diameters wide [14]. Hence, molecular dynamics (MD) simulation is ideally suited to probe the molecular mechanism of phase change. Tsuruta et al. [15] have used molecular dynamics to simulate the stationary evaporation and condensation between two liquid slabs kept at different temperatures and calculated values for the evaporation and condensation coefficients for an argon-like fluid. These coefficients are equal under equilibrium conditions. Anisimov et al. [16] have simulated evaporation into a vacuum by removing all molecules that left the gas 
region of the simulation cell. They have considered a completely absorbing wall, and found the evaporation/condensation coefficient for simple liquids equal to about 0.8 in a wide range of temperatures. Nagayama and Tsuruta $[17,18]$ have studied the condensation coefficients of argon and water based on the transition state theory and molecular dynamics simulation. They found that the condensation coefficients of argon and water decreased when liquid temperatures increased.

Most of the previous studies applied the molecular dynamic approach to the analysis of the evaporation and condensation processes of monatomic or relatively simple polyatomic molecules, such as argon [15], water or methanol $[17,18]$. In our previous paper, the preliminary molecular dynamics simulations have been performed to study the evaporation and condensation of n-dodecane $\left(\mathrm{C}_{12} \mathrm{H}_{26}\right)$ at temperatures in the range 400-600 K [14]. A modified OPLS (Optimized Potential for Liquid Simulation) model has been applied to take into account the Lennard-Jones, bond bending and torsion potentials with the bond length constrained. The equilibrium liquidvapour n-dodecane interface thickness has been predicted to be in the range 1.2-2.0 nm. It has been shown that the molecular chains lie preferentially parallel to the interface in the liquid-vapour transition region. The predicted evaporation/condensation coefficient decreased from 0.9 to 0.3 when temperature increased from 400 $\mathrm{K}$ to $600 \mathrm{~K}$. Typical molecular behaviours in the evaporation and condensation processes have also been presented.

The main aim of this paper is to further investigate the evaporation and condensation processes of $n$ dodecane with particular emphasis on the analysis of the molecular distribution functions in various locations relative to the gas-liquid interface. In Sec. II, the kinetic boundary condition (KBC) is discussed. Sec. III is focused on the presentation of the molecular dynamics models used in simulations of n-dodecane $\left(\mathrm{C}_{12} \mathrm{H}_{26}\right)$ at liquid-vapour quasi-equilibrium state. The analyses of the liquid-vapour quasi-equilibrium system, the mechanism of evaporation and condensation and the velocity distribution function are presented in Sec. IV (the meaning of the term 'quasi-equilibrium' will be discussed in Sec. III). The conclusions are drawn in Sec. V.

\section{KINETIC BOUNDARY CONDITION}

In the kinetic theory, the interface is modelled as a surface of zero thickness $[9,10,13]$, and molecules hitting it from the vapour phase can be captured (condensed) or reflected. Using molecular dynamics simulations for relatively simple molecules, it has been found that the condensation probability does not depend on the incoming velocity $[15,18]$. Also, the incident angle of the molecules has no noticeable effect on the condensation coefficient [18]. 
The distribution function of molecules, leaving the interface from the liquid phase $f^{\text {out }}$ is usually presented as a sum of the distribution functions for the evaporated part $\alpha f^{e}$ and reflected part $(1-\alpha) f^{r}$ [19],

$$
f^{o u t}=\alpha f^{e}+(1-\alpha) f^{r} \quad\left(v_{x}>0\right),
$$

where $\alpha$ is the evaporation/condensation coefficient (these coefficients are equal in the equilibrium state, and assumed to be equal in the quasi-equilibrium state considered in this paper), $f^{e}$ is the distribution function of saturated vapour at the temperature of the condensed phase (liquid) and $v_{x}$ is the velocity component normal to the interface. For evaporated molecules, it is typically assumed that their distribution function is isotropic Maxwellian, with the temperature equal to that of the liquid phase, without drift velocity [20]. The isotropic Maxwellian distributions with drift velocities have been reported in Refs. [15] and [21].

In kinetic modelling, Equation (1) can be considered as the kinetic boundary condition (KBC) [10, 13]. Alternative formulations of the kinetic boundary conditions have been discussed in Refs. [15, 21, 24, 25]. In our previous papers when dealing with heating and evaporation of n-dodecane $\left(\mathrm{C}_{12} \mathrm{H}_{26}\right)$, in Diesel engine-like conditions, we used the kinetic boundary condition (KBC) in the form of Eq. (1) with the evaporation/condensation coefficient set to unity and assuming that the molecular distribution function is isotropic Maxwellian [10-13]. Some authors used Eq. (1) for monatomic molecules of argon [22], and polyatomic molecules of water and methanol [23] in their molecular dynamics simulations.

The maximum mass flux density of the molecules evaporated from the liquid surface, assuming that $f^{e}$ is isotropic Maxwellian, is given by the Hertz formula [26]:

$$
j_{m}=\rho_{v} \sqrt{\frac{k_{B} T_{l}}{2 \pi m}},
$$

where $\rho_{v}$ is the density of the saturated vapour in equilibrium with the liquid at temperature $T_{l}, m$ is the molecular mass and $k_{B}$ is the Boltzmann constant. The ratio of the actual mass flux density of the evaporated molecules $j_{e}$ to its maximum value $j_{m}$ is defined as the evaporation coefficient $\alpha_{\mathrm{e}}$. The condensation coefficient is defined as the ratio of the actual mass flux of condensed molecules $j_{c}$ to $j_{m}$. Under the equilibrium condition, the condensed mass flux should be equal to the evaporated mass flux, i.e. $j_{c}=j_{e}$, which implies that $\alpha_{\mathrm{c}}=\alpha_{\mathrm{e}}=$ $\alpha$. The condensed mass flux $j_{c}$ and the evaporated mass flux $j_{e}$ have been calculated by counting the number of molecules crossing a unit area per unit time at the interface zone.

As discussed in our previous study [14], the molecules of n-dodecane lie preferentially parallel to the interface in the liquid-vapour transition region, instead of being random. Also, it has been found that the translational energies are typically marginally larger than the rotational energies in the region close to the 
interface. Hence the distribution function of evaporating molecules is expected to deviate from the isotropic Maxwellian distribution. This will be discussed in detail in Sec. IV D.

\section{MOLECULAR DYNAMICS SIMULATION}

This section is devoted to the description of the molecular dynamics simulation method and quasiequilibrium simulation system used in present study.

\section{A. Intermolecular potential}

A number of models have been developed to describe the dynamics of complex hydrocarbon molecules, such as n-dodecane $\left(\mathrm{C}_{12} \mathrm{H}_{26}\right)$, including the OPLS (Optimized Potential for Liquid Simulation), originally suggested by Jorgensen et al. [27], and the de Pablo and Toxvaerd models. Smit et al. [28] introduced a new OPLS model, which was claimed to be more accurate comparing with previous models. All these models are based on the observation that the C-H bond in complex hydrocarbon molecules is much shorter and much stronger than the C-C bond, and also stronger than the Van-der-Waals forces between molecules. Thus the methyl $\left(\mathrm{CH}_{3}\right)$ or methylene $\left(\mathrm{CH}_{2}\right)$ groups can be regarded as separate atom-like structures in a relatively simple united atom model (UAM) [29]. The underlying physics of all these models is essentially the same, but they differ by the values of the energy parameters $\varepsilon$ for $\mathrm{CH}_{3}$ and $\mathrm{CH}_{2}$, diameters of these groups, and bond bending and torsion potentials. Smit et al. [28] applied all the above mentioned models to complex hydrocarbons to determine their vapour-liquid coexistence curves, using the Gibbs-ensemble technique and the configuration-bias Monte Carlo method. All models gave nearly identical results at standard conditions but predicted critical temperatures which could differ by up to $100 \mathrm{~K}$ (the critical temperature is mainly controlled by the ratio $\varepsilon_{\mathrm{CH}_{3}} / \varepsilon_{\mathrm{CH}_{2}}$ ). Simon et al. [29] used the de Pablo and Toxvaerd models to study the thermal flux through a surface of n-octane. Based on molecular dynamics simulations, interfacial properties of n-alkane liquid-vapour interfaces had also been studied [30-36].

The molecular dynamics simulation of n-dodecane $\left(\mathrm{C}_{12} \mathrm{H}_{26}\right)$ in an equilibrium system has been also performed using AMBER version 10.0 (a package of molecular simulation programmes) [37]. In that paper, the general Amber force field (GAFF) has been applied and the potential energy has been presented as a sum of bending, torsion, van der Waals and Coulomb energies. As this molecular simulation package takes the hydrogen atoms explicitly into account, it can be called the all atoms model (AAM). Due to the increased number of interactions taken into account, this "all atoms model” is much more time-consuming compared with 
the united atom model (UAM). Hence, the attractiveness of the UAM model for practical engineering application. It has been successfully used for modelling the phase behaviour of a large number of n-alkanes ranging from pentane $\left(\mathrm{C}_{5} \mathrm{H}_{12}\right)$ to octatetracontane $\left(\mathrm{C}_{48} \mathrm{H}_{98}\right)$ [28]. Our simulation is based on the UAM, and we refer to united atoms as atoms and to molecules as chains. The essence of the united atom model applied to an ndodecane molecule is schematically illustrated in Fig. 1.

The non-bonded interactions between atoms, which belong to either different chains or the same chain but more than four atoms apart (there should be at least 3 atoms between the interacting atoms), are characterized by the truncated Lennard-Jones (L-J) 12-6 potential [38, 39],

$$
u^{L J}\left(r_{i j}\right)=4 \varepsilon_{i j}\left[\left(\frac{\sigma_{i j}}{r_{i j}}\right)^{12}-\left(\frac{\sigma_{i j}}{r_{i j}}\right)^{6}\right] .
$$

The energy parameters of $\mathrm{CH}_{2}$ and $\mathrm{CH}_{3}$ groups (atoms) are $\varepsilon_{\mathrm{CH}_{2}} / k_{B}=47 \mathrm{~K}$ and $\varepsilon_{C_{3}} / k_{B}=114 \mathrm{~K}$, respectively. According to the geometric rule, the energy parameter between $\mathrm{CH}_{2}$ and $\mathrm{CH}_{3}$ groups is estimated as $\varepsilon_{\mathrm{CH}_{2}}-\mathrm{CH}_{3} /$ $k_{B}=\sqrt{\varepsilon_{C_{2}} \varepsilon_{C H_{3}}} / k_{B}=73.2 \mathrm{~K}$. The diameters of the methylene and methyl groups are assumed to be equal and estimated as $\sigma_{i j}=\sigma=3.93 \times 10^{-10} \mathrm{~m}$. The L-J $12-6$ interaction is truncated at $13.8 \times 10^{-10} \mathrm{~m}$ in our simulations.

The interactions within the chains include bond bending and torsion with the bond length constrained at $1.53 \times 10^{-10} \mathrm{~m}$. The bending can take place between any three neighbouring atoms. For the bond bending between the three atoms, the van der Ploeg and Berendsen potential is used [40]:

$$
u^{\text {bend }}(\theta)=\frac{1}{2} k_{\theta}\left(\theta-\theta_{0}\right)^{2}
$$

where the bending coefficient is estimated as $k_{\theta} / k_{B}=62500 \mathrm{~K} / \mathrm{rad}^{2}$, and the equilibrium angle is $\theta_{0}=114^{\circ}$ (see Fig. 1). The torsion can take place between four neighbouring atoms. For the torsion potential between the two atoms with two atoms between them, the Jorgensen et al. torsion potential is applied [27]:

$$
u^{\text {tors }}(\varnothing)=c_{0}+0.5 c_{1}(1+\cos \emptyset)+0.5 c_{2}(1-\cos 2 \varnothing)+0.5 c_{3}(1+\cos 3 \emptyset) \text {, }
$$

where $c_{0} / k_{B}=0 \mathrm{~K}, c_{1} / k_{B}=355 \mathrm{~K}, c_{2} / k_{B}=-68.19 \mathrm{~K}, c_{3} / k_{B}=791.3 \mathrm{~K}$ and $\emptyset$ is the dihedral angle. Its equilibrium value is $180^{\circ}$.

\section{B. Equilibrium simulation of a liquid-vapour system}

In the present work, a system of 720 n-dodecane $\left(\mathrm{C}_{12} \mathrm{H}_{26}\right)$ molecules $\left(8640 \mathrm{CH}_{3}\right.$ and $\mathrm{CH}_{2}$ groups) is considered in a three-dimensional rectangular simulation box of $L_{x} \times L_{y} \times L_{z}=64.24 \sigma \times 16.48 \sigma \times 16.48 \sigma$, where $\sigma$ is the rescaled length given in Table I, and $L_{x}, L_{y}$ and $L_{z}$ are the lengths of the simulation box in the $x$-, 
$y$ - and z-directions, for liquid temperatures $400 \mathrm{~K}, 450 \mathrm{~K}, 500 \mathrm{~K}$ and $550 \mathrm{~K}$. This size of box corresponds to $25.25 \mathrm{~nm} \times 6.48 \mathrm{~nm} \times 6.48 \mathrm{~nm}$. These molecules were initially oriented along the $\mathrm{x}$-axis and placed in the middle of the simulation box (see Fig. 2(a)). They had zigzag configurations (see Fig. 1), and the numbers of molecules in the $x$-, $y$ - and z-directions were taken to be 5,12 and 12, respectively.

The equations of motion of the atoms were integrated using the Verlet leapfrog method [38, 39]. For ndodecane chains, the bond length constraints refer only to the near-neighbour atoms, and the bond lengths are constrained by the SHAKE scheme adjusting the atom coordinates one by one cyclically to satisfy a given tolerance [38, 39, 41]. The time step in all simulations was taken equal to $0.002 \tau$, which corresponds to 5 fs. Periodic boundary conditions were applied in all directions. To achieve higher calculation efficiency, reduced units were used for most physical parameters as indicated by the superscript “*” in Table I.

The system was relaxed with a constraint of fixed homogeneous and isotropic temperature. The molecules began to relocate within the liquid phase and to evaporate gradually. We monitored the system temperature, pressure, density, molecular configuration, and interface locations to determine whether or not the system had reached quasi-equilibrium. Typically this required 15000 ps. The term 'quasi-equilibrium' is used following [18] to indicate a state reasonably close to equilibrium, although we are not able to quantify the closeness of this state to the perfect equilibrium state. At the quasi-equilibrium state, the liquid film was sandwiched between the layers of the vapour phases (see Fig. 2(b), (c), (d) and (e) for temperatures $400 \mathrm{~K}, 450 \mathrm{~K}, 500 \mathrm{~K}$ and $550 \mathrm{~K}$ respectively). The densities in the liquid and vapour zones gradually approached to those of actual liquid and vapour. Then we started sampling data for another 10000 ps. The positions of the two liquid-vapour interfaces were identified by the density profiles. The interface parameters, such as density and evaporation/condensation coefficient, were obtained by averaging them over these 10000 ps. The effects of hydrodynamic turbulence have not been taken into account in our analysis.

The system with 720 molecules enabled us to perform the molecular dynamics simulations using a standard PC (2.83 GHz CPU, 4 GB RAM work station) over a reasonable time period (just over one week per simulation).

\section{SIMULATION RESULTS}

In this section, simulation results referring to the liquid-vapour quasi-equilibrium state are presented and discussed. 


\section{A. Liquid-vapour phase quasi-equilibrium at the interface}

Quasi-equilibrium density profiles at temperatures $400 \mathrm{~K}, 450 \mathrm{~K}, 500 \mathrm{~K}$ and $550 \mathrm{~K}$ are shown in Fig. 3. These profiles are time-averaged over 5000 ps during a steady state period. As follows from this figure, at higher liquid temperatures, the densities of the liquid phase are lower, as expected. The liquid-vapour coexistence curve is shown in Fig. 4, and the densities of vapour and liquid phases are presented in Table II. As follows from this figure, our results agree reasonably well with experimental data [42] and the results of the Monte Carlo simulations [28]. The predicted densities of the liquid phase are marginally higher than the ones predicted by the Monte Carlo method; our results referring to the vapour phase are in marginally better agreement with experimental data than the Monte Carlo results. The critical temperature and density are estimated to be about $720 \mathrm{~K}$ and $45 \mathrm{~kg} / \mathrm{m}^{3}$, respectively, which are reasonably consistent with the experimental data: $657.2 \mathrm{~K}$ and $56.85 \mathrm{~kg} / \mathrm{m}^{3}$. Note that in [24] the OPLS potential was used to simulate the evaporation of methanol; the estimated critical temperature was more than $100 \mathrm{~K}$ lower than that obtained experimentally. The results shown in Figs. 3 and 4 are more accurate than those presented in Figs. 4 and 5 in [14] due to the larger number of molecules used in our simulation.

The thickness of the transition layer at the liquid-vapour interface (interface thickness) is defined as the thickness of the region where the bulk vapour phase changes to the bulk liquid phase. In Fig. 3, this refers to the thickness over which the density of n-dodecane changes from $0.95 \rho_{l}$ to $\left(\rho_{v}+0.01 \rho_{l}\right)$, where $\rho_{l}$ and $\rho_{v}$ are densities of liquid and vapour, respectively [18]. The calculated values of the interface thickness of n-dodecane, presented in Table II, versus reduced temperatures are shown in Fig. 5. In the same figure, the interface thicknesses for argon, water and methanol, reported in Refs. [18] and [23], are also presented. As one can see in Fig. 5, the thickness of the transition layer of n-dodecane increases from about 2.25 to $3.5 \mathrm{~nm}$ when the reduced temperature increases from about 0.6 to about 0.85 , which corresponds to temperatures increased from 400 to $550 \mathrm{~K}$. These values are noticeably larger than those of argon [18], water [18] and methanol [24] (note that critical temperatures for argon, water and methanol are $150.86 \mathrm{~K}, 647.13 \mathrm{~K}$ and $403.73 \mathrm{~K}$, respectively). The greater thickness of the transition layer at high temperatures may reduce the evaporation/condensation probability of n-dodecane $\left(\mathrm{C}_{12} \mathrm{H}_{26}\right)$ molecules at the liquid surface. For chain-like n-dodecane $\left(\mathrm{C}_{12} \mathrm{H}_{26}\right)$ molecules, the large thickness of the transition layer would act as a barrier to the condensation of vapour molecules or the evaporation of molecules leaving the liquid surface. Hence, it is expected that the increased thickness of the transition layer at high temperatures would result in smaller evaporation/condensation 
coefficients. This is confirmed by our estimates of the evaporation/condensation coefficient presented later in this paper.

\section{B. Evaporation and condensation behaviours}

The mechanism of evaporation and condensation of $n$-dodecane $\left(\mathrm{C}_{12} \mathrm{H}_{26}\right)$ has been studied in detail by recording the trajectories and translational energies of molecules. Examples are shown in Figs. 6-9. As follows from Fig. 6, a molecule escapes from the bulk of the liquid and is evaporated (see Fig. 6(a)) when its translational energy abruptly increases (see Fig. 6(b)). In the case shown in Fig. 7, the condensation of a molecule is accompanied by the reduction of its translational energy (see Fig. 7(b)). This energy in the liquid phase remains close to the mean kinetic energy in the system. In the case shown in Fig. 8, a molecule gathers large translational energy and moves outward from the bulk of the liquid towards the interface; during this process, it collides with other molecules and loses most of its translational energy before it reaches the interface (see Fig. 8(b)). As a result, the molecule returns to the liquid phase (see Fig. 8(a)). In the case shown in Fig. 9, the molecule with large translational energy in the liquid phase enters the vapour phase and then it returns to the interface (see Fig. 9(a)). Then it collides with other molecules at the interface and returns into the vapour phase losing most of its translational energy (see Fig. 9(b)). The evaporation and condensation behaviours shown in Figs. 6-9 allow us to conclude that the translational energy plays an important role in the evaporation and condensation processes. Note that Figs. 6-9 are complementary to Fig. 10 in [14], where a number of trajectories of molecules are presented although without the accompanying plots for the translational energies.

The effect of the translational energy on the evaporation and condensation processes of n-dodecane $\left(\mathrm{C}_{12} \mathrm{H}_{26}\right)$ is similar to its effect on the monatomic molecule of argon [15] and polyatomic molecules of water and methanol [17]. This supports the idea of activation energy in the liquid-vapour transition, which follows from the transition state theory [17]. The transition layer builds a three dimensional structure at the liquid-vapour interface with a kind of interfacial resistance as discussed in Sec. IV A. The fact that the molecules in the transition layer preferentially lie parallel to the interface is expected to contribute to the blocking of molecular evaporation and condensation.

\section{Evaporation/condensation coefficient}

Assuming that the distribution function of molecules at the interface is isotropic Maxwellian (which will be confirmed later by our calculations), the estimation of the maximum flux by Eq. (2) can be justified. Hence, in 
our calculations the evaporation and condensation coefficients have been estimated as the ratios of evaporated and condensed mass fluxes to the maximum fluxes given by Eq. (2) (see Sec. II). The equality of evaporation and condensation coefficients in the equilibrium allows us to focus our analysis on one of these coefficients. The condensation coefficient was chosen. The result was compared with the predictions of the transition state theory [17], in which the condensation coefficient is estimated as:

$$
\alpha_{c}=\left[1-\left(\frac{V^{l}}{V^{g}}\right)^{1 / 3}\right] \exp \left[-\frac{1}{2} \frac{1}{\left(\frac{V^{g}}{V^{l}}\right)^{1 / 3}-1}\right],
$$

where $V^{l}$ and $V^{g}$ are specific volumes of the liquid and gas. In this theory, condensation and evaporation processes at the liquid-vapour interface are considered as a kind of chemical reaction and the general theory of rate processes is applied [43].

The condensation coefficient of n-dodecane was calculated for liquid temperatures $T_{l}=400 \mathrm{~K}, 450 \mathrm{~K}, 500 \mathrm{~K}$ and $550 \mathrm{~K}$ and the results are shown in Table II. The plots of $\alpha_{\mathrm{c}}$ versus the reduced temperature for n-dodecane (critical temperature 657.2 K) are shown in Fig. 10 (filled circles). As follows from this figure, the condensation coefficient decreases from about 0.93 at $400 \mathrm{~K}$ to about 0.45 at $550 \mathrm{~K}$. The condensation coefficients of ndodecane, reported in [14] using MD simulation in the system with 400 molecules, are also shown in Fig. 10 (open circles). The values of the condensation coefficient predicted by the transition state theory (Eq. (6)) have been obtained using the densities of the liquid and vapour phases obtained in our MD simulations (dotted line). As follows from this figure, there is a general agreement between the present results, the predictions of the transition state theory, and the prediction of [37], although the reasons for noticeable deviation between these results are still to be investigated. One of the reasons could be linked with the limited number of molecules used in our calculations. Also, the united atom model, used in our calculations, might be less accurate at reduced temperatures close to 1 . The condensation coefficients of other substances using MD simulations reported by other authors are also shown in Fig. 10 (filled [15] and open [22] squares for argon, filled [23] and open [18] triangles for water and filled diamonds for methanol [23]).

As follows from Fig. 10, in all cases the condensation coefficient decreases when the liquid temperature increases. This is consistent with the results for the interface thickness shown in Fig. 5. As mentioned earlier, the thicker interfaces at higher temperature are expected to reduce the values of the evaporation/condensation coefficients.

The condensation coefficient for complex polyatomic molecules, like n-dodecane, would be expected to be lower than for simpler molecules due to the constraint imposed by the rotational motion of molecules in the 
liquid phase [44]. However, in our MD simulations, it has been found that the rotational energy has no noticeable effect on this coefficient and its value is mainly controlled by the translational energy in agreement with the previously reported results $[14,18]$. On the other hand, based on the transition state theory, we can expect that this coefficient is close to unity at low temperatures when $\left(V^{g} / V^{l}\right)^{1 / 3}$ is large, and decreases with increasing temperature due to the decrease of the ratio $\left(V^{g} / V^{l}\right)^{1 / 3}$. This trend is consistent with the prediction of our MD simulations.

The values of the evaporation/condensation coefficient predicted by our MD simulations can be used in the formulation of the boundary conditions in kinetic modelling, instead of the previously used assumptions that this coefficient is equal to unity [10-13] or rather arbitrary values 0.04 or 0.5 [9].

\section{Velocity distribution function}

As mentioned in Sec. II, it is generally assumed that the distribution functions of the evaporated molecules and the molecules entering the kinetic region from the hydrodynamic one are isotropic Maxwellian. In what follows the range of applicability of this assumption is assessed based on calculations of this distribution function using the results of our MD simulations. Our analysis is focused on the investigation of this function inside the liquid phase, at the interface and at the vapour phase.

The distribution function of translational velocities of the mass centres of molecules can be calculated as [23]:

$$
f_{j}=\frac{1}{\rho N_{s} \Xi_{p} \Xi_{v}^{j}} \sum_{N_{S}} \sum_{i \in\left(\Xi_{p} \cap \Xi_{v}^{j}\right)} m_{i}
$$

where $f_{j}$ is the distribution of the $j$ component of the translational velocity $\left(f_{j}=f_{x, y, z}\right)$, $\Xi_{p}$ is a volume element in the physical space, $\Xi_{v}^{j}$ is a one-dimensional volume element in the $j$ direction in the three-dimensional molecular velocity space, and $\Xi_{p} \cap \Xi_{v}^{j}$ denotes a four-dimensional volume element in the six-dimensional physical/velocity space, $m_{i}$ are masses of individual molecules, assumed to be the same. The distribution function $f_{j}$ is normalized by the density $\rho$ calculated in the volume element $\Xi_{p}$ and sampled by $N_{s}=20000$. $\Sigma_{N_{s}}$ indicates the summation over $N_{s}$ samples. The summation of $f_{j}$, defined by (7), over $\Xi_{v}^{j}$ gives 1 (this function is normalised by unity).

The velocity distribution function was found by sampling the velocities of molecules in the control volumes of thickness $0.642 \sigma=L_{x} / 100$ in the $x$ direction, in three regions: inside the liquid phase, interface and vapour phase. Alternatively, the velocity distribution function could be found by transforming the differential fluxes [15], but this approach has various numerical problems [21]. It has not been used in our analysis. 
Figs. 11 and 12 show the distributions of molecules for $y$ - and z-velocity components $v_{y}$ and $v_{z}$ (directions parallel to the interface) in the above mentioned three regions at two temperatures $500 \mathrm{~K}$ and $550 \mathrm{~K}$. The corresponding Maxwellian distributions for the same temperatures are shown in these figures by solid curves. As one can see from these figures, despite a considerable scatter of data in the vapour phase, for both temperatures and in all three regions the distributions of tangential velocity components are close to isotropic Maxwellian. Figs. 13 and 14 show the distributions of evaporated molecules for the $x$-velocity components $v_{x}$ (the direction normal to the interface) for the same temperatures as in Figs. 11 and 12. The corresponding Maxwellian distributions for the same temperatures as in the case shown in Figs. 11 and 12 are shown in these figures by solid curves. As can be seen from these figures, in the liquid phase and in the interface, the predicted distribution functions are close to isotropic Maxwellian, corresponding to temperatures $500 \mathrm{~K}$ and $550 \mathrm{~K}$, as in the cases shown in Figs. 11 and 12. The velocity distributions in the vapour phase, however, turned out to be rather different from the above mentioned Maxwellian distributions. These distributions are close to Maxwellian, but with temperatures higher than in the liquid phase and the interface. The latter are shown in Figs. 13 and 14 by dotted curves for temperatures for $627 \mathrm{~K}$ and $681 \mathrm{~K}$, respectively. These temperatures were shown to provide the best fit to the predicted distribution of molecules. Thus, the distribution of molecules in the vapour phase is expected to be close to bi-Maxwellian with different temperatures in the direction normal to the interface. This means that the evaporated molecules have larger average translational energies in the direction perpendicular to the interface than the corresponding energies in the direction parallel to the interface. Note that these distributions are typical for the outer space plasma [45].

The bi-Maxwellian distribution of molecules in the gas phase indicates that gas in this region is not in an equilibrium state. This does not contradict the analysis presented in [46], where the region in the gas phase close to the interface is explicitly called the 'nonequilibrium region'. We should admit, however, that further studies of the problem, using larger numbers of molecules, are required to predict a more reliable shape of the distribution function in this region.

These results are consistent with the results reported earlier in Sec. II and our previous study [14]. The same phenomenon has also been observed in the MD simulations of argon [15, 21]. Although we appreciate that this result needs to be confirmed by MD simulations with much larger numbers of molecules, we believe that the traditional approach to the formulation of the boundary conditions in kinetic modelling, when the distribution function of the evaporated molecules is assumed to be isotropic Maxwellian with the same temperatures for all velocity components, needs to be investigated more carefully. 


\section{CONCLUSIONS}

The evaporation and condensation of n-dodecane $\left(\mathrm{C}_{12} \mathrm{H}_{26}\right)$, the closest approximation to Diesel fuel, have been investigated using the molecular dynamics (MD) simulation technique. The interactions in chain-like molecular structures are modelled using optimised potential for liquid simulations (OPLS). The thickness of the transition layer between liquid and vapour phases at equilibrium is estimated to be $2.25-3.5 \mathrm{~nm}$. It has been pointed out that the molecules at the liquid surface need to have relatively large translational energy to evaporate. The vapour molecules with large translational energy can easily penetrate deeply into the transition layer and condense in the liquid phase. The values of the evaporation/condensation coefficient at various liquid phase temperatures have been estimated. It has been demonstrated that this coefficient decreases with temperature, in agreement with the prediction of the transition state theory. The molecular velocity distribution functions in the liquid phase and the interface have been shown to be close to isotropic Maxwellian with temperatures equal to the one of the liquid phase. These functions in the vapour phase, however, have been shown to deviate from the classical isotropic Maxwellian distributions. They can be approximated as bi-Maxwellian, with temperatures in the direction normal to the interface larger than those in the directions parallel to the interface.

\section{ACKNOWLEDGEMENTS}

The authors are grateful to EPSRC (grant EP/H001603/1) of the UK, the National Natural Science Foundation of China (grant 50976052), and the Cross-discipline Foundation of the Tsinghua National Laboratory for Information Science and Technology (TNList) for their financial support of this project.

\section{RERERENCES}

[1] E.E. Michaelides, "Hydrohynamic force and heat/mass transfer from particles, bubbles, and drops-the Freeman scholar lecture,“ ASME J. Fluid Eng. 125, 209 (2003).

[2] U. Fritsching, Spray simulation (Cambridge University Press, Cambridge, England, 2004).

[3] S.S. Sazhin, "Advanced models of fuel droplet heating and evaporation,“ Prog. Energy Combust. Sci. 32, 162 (2006).

[4] A. Faghri and Y. Zhang, Transport phenomena in multiphase systems (Elsevier, New York, 2006).

[5] E.E. Michaelides, Particles, Bubbles \& Drops (World Scientific, Singapore, 2006).

[6] S.S. Sazhin, P.A. Krutitskii, I.G. Gusev, and M.R. Heikal, "Transient heating of an evaporating 
droplet,“ Int. J. Heat Mass Transf. 53, 2826 (2010).

[7] S.S. Sazhin, P.A. Krutitskii, I.G. Gusev, and M.R. Heikal, "Transient heating of an evaporating droplet with presumed time evolution of its radius,“ Int. J. Heat Mass Transf. 54, 1278 (2011).

[8] S.S. Sazhin, A. Elwardany, P.A. Krutitskii, V. Depredurand, G. Castanet, F. Lemoine, E.M. Sazhina, and M.R. Heikal, "Multi-component droplet heating and evaporation: Numerical simulation versus experimental data,“ Int. J. Therm. Sci. 50, 1164 (2011).

[9] A.P. Kryukov, V.Y. Levashov, and S.S. Sazhin, "Evaporation of diesel fuel droplets: kinetic versus hydrodynamic models,“ Int. J. Heat Mass Transf. 47, 2541 (2004).

[10] S.S. Sazhin, I.N. Shishkova, A.P. Kryukov, V.Yu. Levashov, and M.R. Heikal, "Evaporation of droplets into a background gas: kinetic modelling,“ Int. J. Heat Mass Transf. 50, 2675 (2007).

[11] S.S. Sazhin and I.N. Shishkova, "A kinetic algorithm for modelling the droplet evaporation process in the presence of heat flux and background gas,“ Atomization Sprays 19, 473 (2009).

[12] S.S. Sazhin, I.N. Shishkova, and M. Heikal, "Kinetic modelling of fuel droplet heating and evaporation: calculations and approximations,“ Int. J of Eng. Sys. Model. Simul. 2, 169 (2010).

[13] I. N. Shishkova and S.S. Sazhin, “A numerical algorithm of kinetic modelling of evaporation processes,“ J. Comput. Phys. 218, 635 (2006).

[14] B.-Y. Cao, J.-F. Xie, and S.S. Sazhin, "Molecular dynamics study on evaporation and condensation of ndodecane at liquid-vapour phase equilibria,“ J. Chem. Phys. 134, 164309 (2011).

[15] T. Tsuruta, H. Tanaka, and T. Masuoka, "Condensation/evaporation coefficient and velocity distributions at liquid-vapour interface,“ Int. J. Mass Heat Transf. 42, 4107 (1999).

[16] S.I. Anisimov, D.O. Dunikov, V.V. Zhakhovskii, and S.P. Malyshenko, "Properties of a liquid-vapor interface at high-rate evaporation,“ J. Chem. Phys. 110, 8722 (1999).

[17] G. Nagayama and T. Tsuruta, “A general expression for the condensation coefficient based on transition state theory and molecular dynamics simulation,“ J. Chem. Phys. 118, 1392 (2003).

[18] T. Tsuruta and G. Nagayama, "Molecular dynamics studies on the condensation coefficient of water,“ J. Phys. Chem. B 108, 1736 (2004).

[19] C. Cercignani, The Boltzmann Equation and Its Application (Springer-Verlag, Berlin, 1988).

[20] R. Meland, Molecular exchange and its influence on the condensation coefficient, J. Chem. Phys. 117 (2002), 7254-7258.

[21] R. Meland and T. Ytehus, "Boundary condition at a gas-liquid interphase,“ in 22th Internaltional 
Sysposium of Rarefied Gas Dynamics, edited by T.J. Bartel and M.A. Gallis, AIP Conf. Pro. No. 585 (AIP, Melville, 2001), p. 583.

[22] T. Ishiyama, T. Yano, and S. Fujikawa, "Molecular dynamics study of kinetic boundary conditions at an interface between argon vapor and its condensed phase,“ Phys. Fluids 16, 2899 (2004).

[23] T. Ishiyama, T. Yano, and S. Fujikawa, "Molecular dynamics study of kinetic boundary conditions at an interface between a polyatomic vapor and its condensed phase,“ Phys. Fluids 16, 4713 (2004).

[24] T. Ishiyama, T. Yano, and S. Fujikawa, "Kinetic boundary condition at a vapour-liquid interface,“ Phys. Rev. Lett. 95, 084504 (2005).

[25] A. Frezzotti, "Boundary condition at the vapor-liquid interface,“ Phys. Fluids 23, 030609 (2011).

[26] L.D. Landau and E.M. Lifshitz, Statistical Physics, Pt. 1, 3rd. ed. (Pergamon Press, Oxford, 1980).

[27] W.L. Jorgensen, J.D. Madura, and C.J. Swenson, “Optimized intermolecular potential functions for liquid hydrocarbons,“ J. Am. Chem. Soc. 106, 6638 (1984).

[28] B. Smit, S. Karaborni, and J.I. Siepmann, "Computer simulations of vapour-liquid phase equilibria of nalkanes,“ J. Chem. Phys. 102, 2126 (1995).

[29] J.M. Simon, S. Kjelstrup, D. Bedeaux, and B. Hafskjold, "Thermal flux through a surface of n-octane. A non-equilibrium molecular dynamics study,“ J. Phys. Chem. 108, 7186 (2004).

[30] J.G. Harris, "Liquid vapour interface of alkane oligomers-structure and thermodynamics from moleculardynamics simulations of chemically realistic models,“ J. Phys. Chem. 96, 5077 (1992).

[31] J.M. Simon, D. Bedeaux, S. Kjelstrup, J. Xu, and E. Johannessen, "Interface film resistivities for heat and mass transfers - integral relations verified by non-equilibrium moilecular dynamics, “ J. Phys. Chem. 110, 18528 (2006).

[32] C. Ibergay, A. Ghoufi, F. Goujon, P. Ungerer, A. Boutin, B. Rousseau, and P. Malfreyt, "Molecular simulations of the n-alkane liquid-vapour interface interfacial properties and their long range corrections,“ Phys. Rev. E 75, 051602 (2007).

[33] Jialin Ge, S. Kjelstrup, D. Bedeaux, J. M. Simon, and B. Rousseau, “Transfer coefficient for evaporation of a system with a Lennard-Jones long-range spline potential,” Phys. Rev. E 75, 061604 (2007).

[34] D. Zahn, "Length-dependent nucleation mechanism rule the vapourization of n-alkane," Chem. Phys. Lett. 467, 80 (2008).

[35] K. Bucior, L. Yelash, and K. Binder, "Molecular-dynamics simulation of evaporation processes of fluid bridges confined in slitlike pores,” Phys. Rev. E 79, 031604 (2009). 
[36] M.A. Amat and G.C. Rutledge, "Liquid-vapour equilibria and interfacial properties of n-alkanes and perfluoroalkanes by molecular simulation,“ J. Chem. Phys. 132, 114704 (2010).

[37] H. Mizuguchi, G. Nagayama, and T. Tsuruta, "Molecular dynamics study on evaporation coefficient of biodiesel fuel,“ in Seventh International Conference on Flow Dynamics, (Sendai, Japan, 2010), p. 386.

[38] M.P. Allen and D.J. Tildesley, Computer Simulation of Liquids (Clarendon, Oxford, 1987).

[39] D.C. Rapaport, The Art of Molecular Dynamics Simulation (Cambridge University Press, Cambridge, England, 1995).

[40] P. Van der Ploeg and H.J.C. Berendsen, “Molecular dynamics simulation of a bilayer membrane,“ J. Chem. Phys. 76, 3271 (1982).

[41] J.P. Ryckaert, G. Ciccotti, and H.J.C. Berendsen, "Numerical integration of the cartesian equations of motion of a system with constraints: molecular dynamics of n-alkanes,“ J. Comput. Phys. 23, 327 (1977).

[42] J.B. Maxwell, Data Book on Hydrocarbons: Application to Process Engineering (Van Nostrand, Princeton, NJ, 1955).

[43] G. Gladstone, K.J. Laidler, and H. Eyring, The Theory of Rate Processes (McGraw-Hill, New York, 1941).

[44] S. Fujikawa and M. Maerefat, “A study of the molecular mechanism of vapor condensation,“ JSME Int. J. Series II 33, 634 (1990).

[45] S.S. Sazhin, Whistler-Mode Waves in a Hot Plasma (Cambridge University Press, 1993).

[46] S. Fujikawa, T. Yano and M. Watanabe, Vapor-Liquid Interfaces, Bubbles and Droplets (Springer, Heidelberg, 2011)

Table I. Values of normalisation parameters used in the MD simulations. 


\begin{tabular}{ll}
\hline Parameters & Values \\
\hline Mass $\left(m^{*}\right)$ & $m_{C_{H_{2}}}=2.3252 \times 10^{-26} \mathrm{~kg}$ \\
Energy $\left(\varepsilon^{*}\right)$ & $\varepsilon_{C H_{2}}=0.6486 \times 10^{-21} \mathrm{~J}$ \\
Length $\left(l^{*}\right)$ & $\sigma=3.93 \times 10^{-10} \mathrm{~m}$ \\
Temperature $\left(T^{*}\right)$ & $\varepsilon_{C H_{2}} / k_{B}=47 \mathrm{~K}$ \\
Density $\left(\rho^{*}\right)$ & $m_{C H_{2}} / \sigma^{3}=382.961 \mathrm{~kg} / \mathrm{m}^{3}$ \\
Time $\left(t^{*}\right)$ & $\tau=\sigma \sqrt{\left(\mathrm{m} / \varepsilon_{C H_{2}}\right)}=2.353 \times 10^{-12} \mathrm{~s}$ \\
Velocity $\left(v^{*}\right)$ & $\sigma / \tau=167 \mathrm{~m} / \mathrm{s}$ \\
\hline
\end{tabular}

Table II. The results of liquid-vapour equilibrium simulations for n-dodecane $\left(\mathrm{C}_{12} \mathrm{H}_{26}\right) \cdot T_{l}$ is liquid temperature, $\rho_{v}$ is saturated vapour density, $\rho_{l}$ is liquid density, $p_{v}$ is vapour pressure, $\delta$ is thickness of the interface, $j_{c}$ is mass flux density of condensed molecules, $j_{m}$ is the maximum mass flux density of molecules evaporated from the liquid surface, estimated based on Eq. (2), and $\alpha_{c}$ is the condensation coefficient.

\begin{tabular}{cccccccc}
\hline$T_{l}[\mathrm{~K}]$ & $\rho_{v}\left[\mathrm{~kg} / \mathrm{m}^{3}\right]$ & $\rho_{l}\left[\mathrm{~kg} / \mathrm{m}^{3}\right]$ & $p_{v}[\mathrm{~atm}]$ & $\delta\left[10^{-9} \mathrm{~m}\right]$ & $j_{c}\left[\mathrm{~kg} /\left(\mathrm{m}^{2} \mathrm{~s}\right)\right]$ & $j_{m}\left[\mathrm{~kg} /\left(\mathrm{m}^{2} \mathrm{~s}\right)\right]$ & $\alpha_{c}$ \\
\hline 400 & 2.823 & 736.374 & 0.544 & 2.27 & 141.63 & 157.37 & 0.93 \\
450 & 5.647 & 647.831 & 1.224 & 2.78 & 230.38 & 333.88 & 0.72 \\
500 & 9.737 & 615.466 & 2.345 & 3.28 & 358.04 & 606.85 & 0.59 \\
550 & 12.376 & 572.025 & 3.279 & 3.53 & 364.04 & 808.97 & 0.45 \\
\hline
\end{tabular}




\section{Figure Captions}

FIG. 1. Schematic presentations of the molecular structure of n-dodecane $\left(\mathrm{C}_{12} \mathrm{H}_{26}\right)$ (a) and its approximation using the united atom model (UAM) including methyl $\left(\mathrm{CH}_{3}\right)$ and methylene $\left(\mathrm{CH}_{2}\right)$ groups (b). The bending angles between neighbouring bonds $\left(\sim 114^{\circ}\right)$ (zigzag structure of the molecule) are shown.

FIG. 2. Snapshots of the simulation box $64.24 \sigma \times 16.48 \sigma \times 16.48 \sigma$ : initial state (a) and liquid-vapour quasiequilibrium state at $400 \mathrm{~K}$ (b), $450 \mathrm{~K}$ (c), $500 \mathrm{~K}$ (d) and $550 \mathrm{~K}(\mathrm{e})$, respectively.

FIG. 3. Density profiles at four temperatures as predicted by present molecular dynamics (MD) simulations.

FIG. 4. Liquid-vapour coexistence results in the equilibrium state as predicted by the present molecular dynamics simulation, Monte Carlo simulations and observed experimentally.

FIG. 5. The thickness of the liquid-vapour interface versus reduced liquid temperature, as predicted by the present molecular dynamics (MD) simulations for n-dodecane, and inferred from the published results for argon, water and methanol.

FIG. 6. A typical time history of a spontaneously evaporating n-dodecane molecule at $500 \mathrm{~K}$ : (a) trajectory of a molecule in the physical space; (b) time evolution of its translational energy (normalized by $\varepsilon_{\mathrm{CH}_{2}}$ given in Table I).

FIG. 7. A typical time history of a spontaneously condensing n-dodecane molecule at $500 \mathrm{~K}$ : (a) trajectory of a molecule in the physical space; (b) time evolution of its translational energy (normalized by $\varepsilon_{\mathrm{CH}_{2}}$ given in Table I).

FIG. 8. A typical time history of an n-dodecane molecule in the liquid phase at $500 \mathrm{~K}$ : (a) trajectory of a molecule in the physical space; (b) time evolution of its translational energy (normalized by $\varepsilon_{\mathrm{CH}_{2}}$ given in Table I).

FIG. 9. A typical time history of an evaporating n-dodecane molecule at $500 \mathrm{~K}$ : (a) trajectory of a molecule in the physical space; (b) time evolution of its translational energy (normalized by $\varepsilon_{\mathrm{CH}_{2}}$ given in Table I).

FIG. 10. The predicted values of the condensation coefficient versus reduced temperature as predicted by the present molecular dynamics (MD) simulation for n-dodecane, inferred from published data for ndodecane, argon, water and methanol, and predicted by the transition state theory.

FIG. 11. The distribution function of molecules with respect to the velocity components tangential to the interface $v_{y}$ (a) and $v_{z}$ (b) in the liquid phase, interface and the vapour phase as predicted by the present molecular dynamics simulations for liquid temperature equal to $500 \mathrm{~K}$. The solid curve shows a onedimensional normalised Maxwellian distribution at $500 \mathrm{~K}$. 
FIG. 12. The same as Fig. 11 but for liquid temperature equal to $550 \mathrm{~K}$. The solid curve shows a onedimensional normalised Maxwellian distribution at $500 \mathrm{~K}$.

FIG. 13. The distribution function of molecules with respect to the velocity component normal to the interface $v_{x}(\mathrm{a})$ in the liquid phase, interface and the vapour phase as predicted by the present molecular dynamics simulations for liquid temperature equal to $500 \mathrm{~K}$. The solid and dotted curves show onedimensional normalised Maxwellian distributions at $500 \mathrm{~K}$ and $627 \mathrm{~K}$ respectively.

FIG. 14. The same as Fig. 13 but for liquid temperature equal to $550 \mathrm{~K}$. The solid and dotted curves show onedimensional normalised Maxwellian distributions at $550 \mathrm{~K}$ and $681 \mathrm{~K}$ respectively. 
Figures

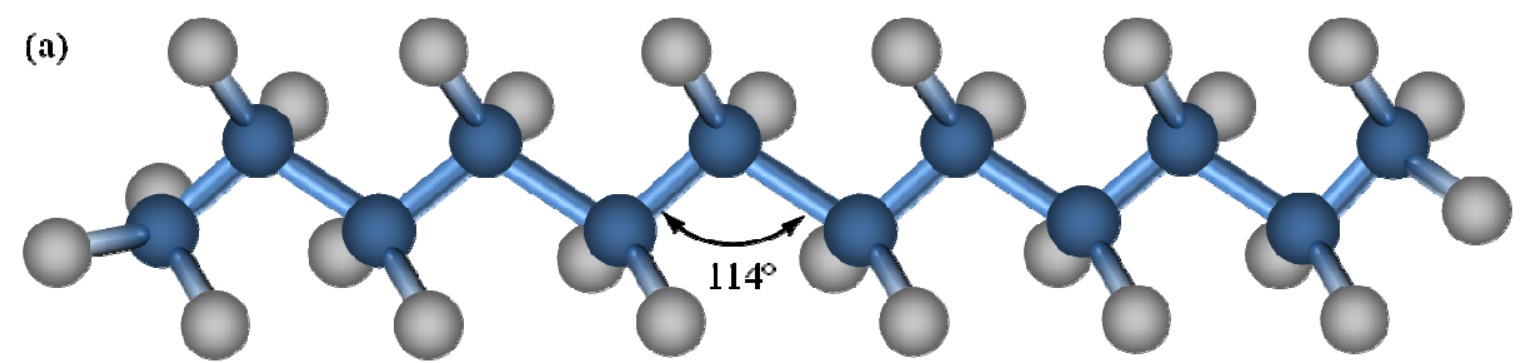

Hydrogen atom Carbon atom

(b)

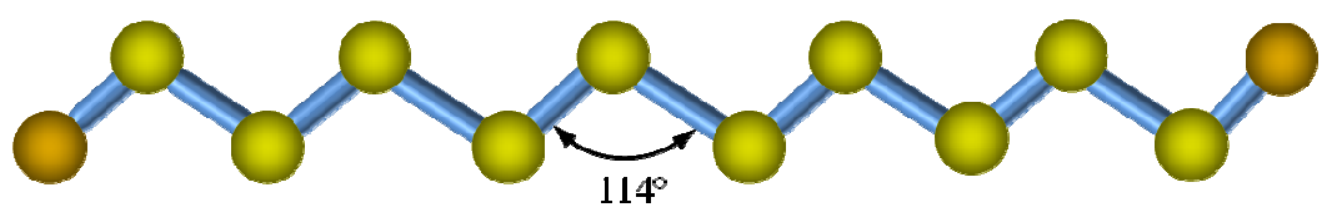

Methyl group $\left(\mathrm{CH}_{3}\right)$

Methylene group $\left(\mathrm{CH}_{2}\right)$

Fig. 1 
(a)

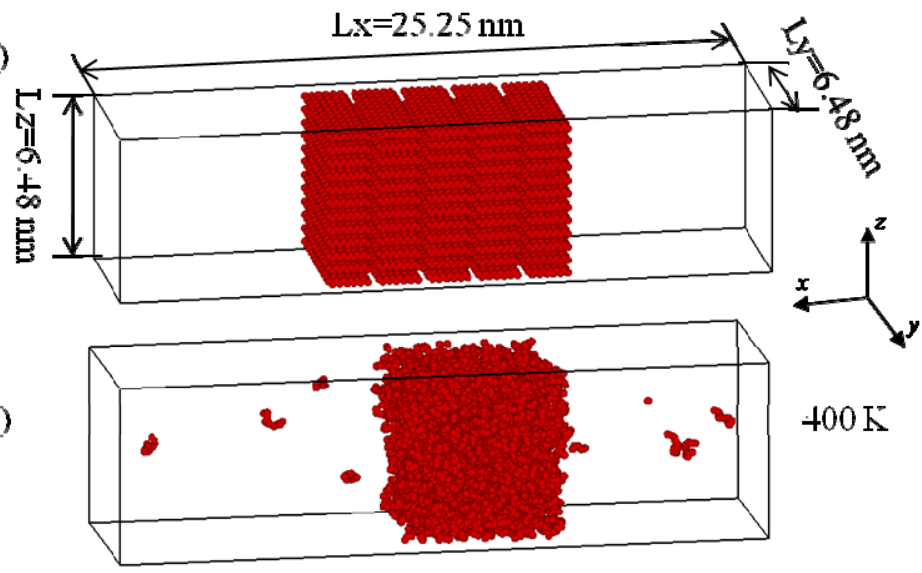

(c)

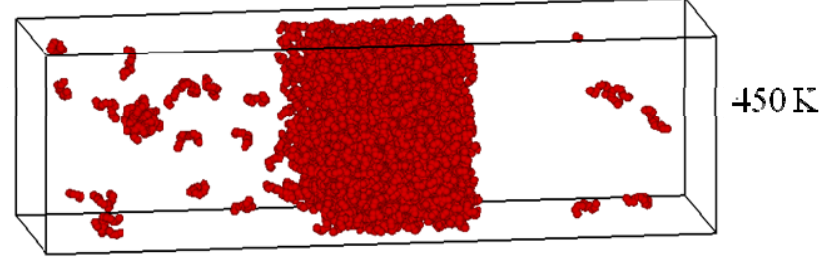

(d)

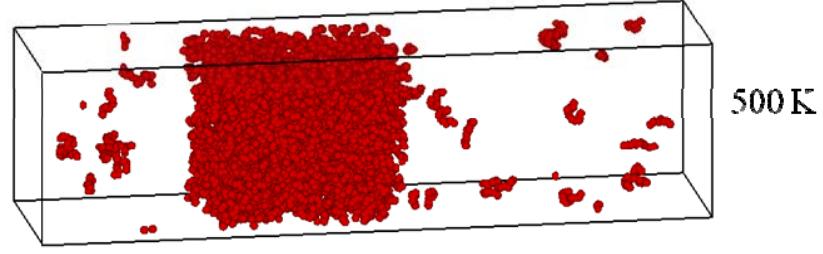

(e)

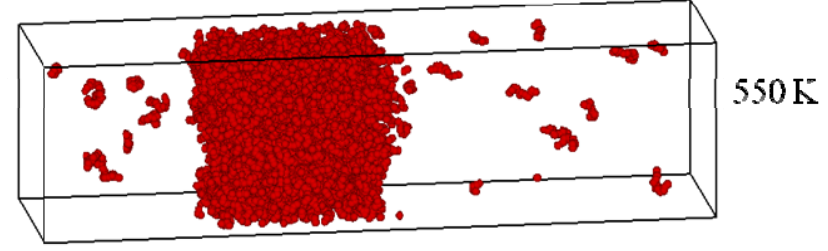

Fig. 2 


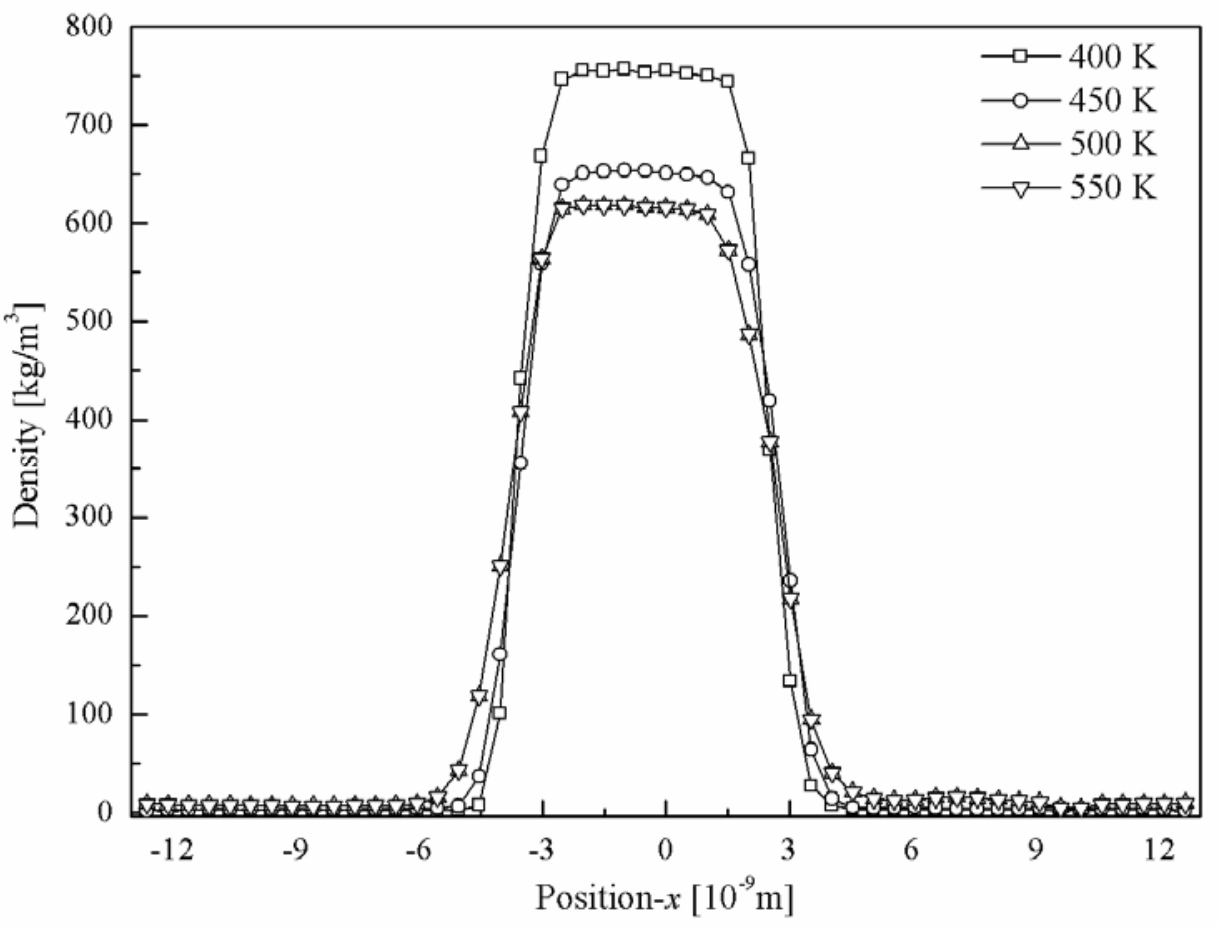

Fig. 3 


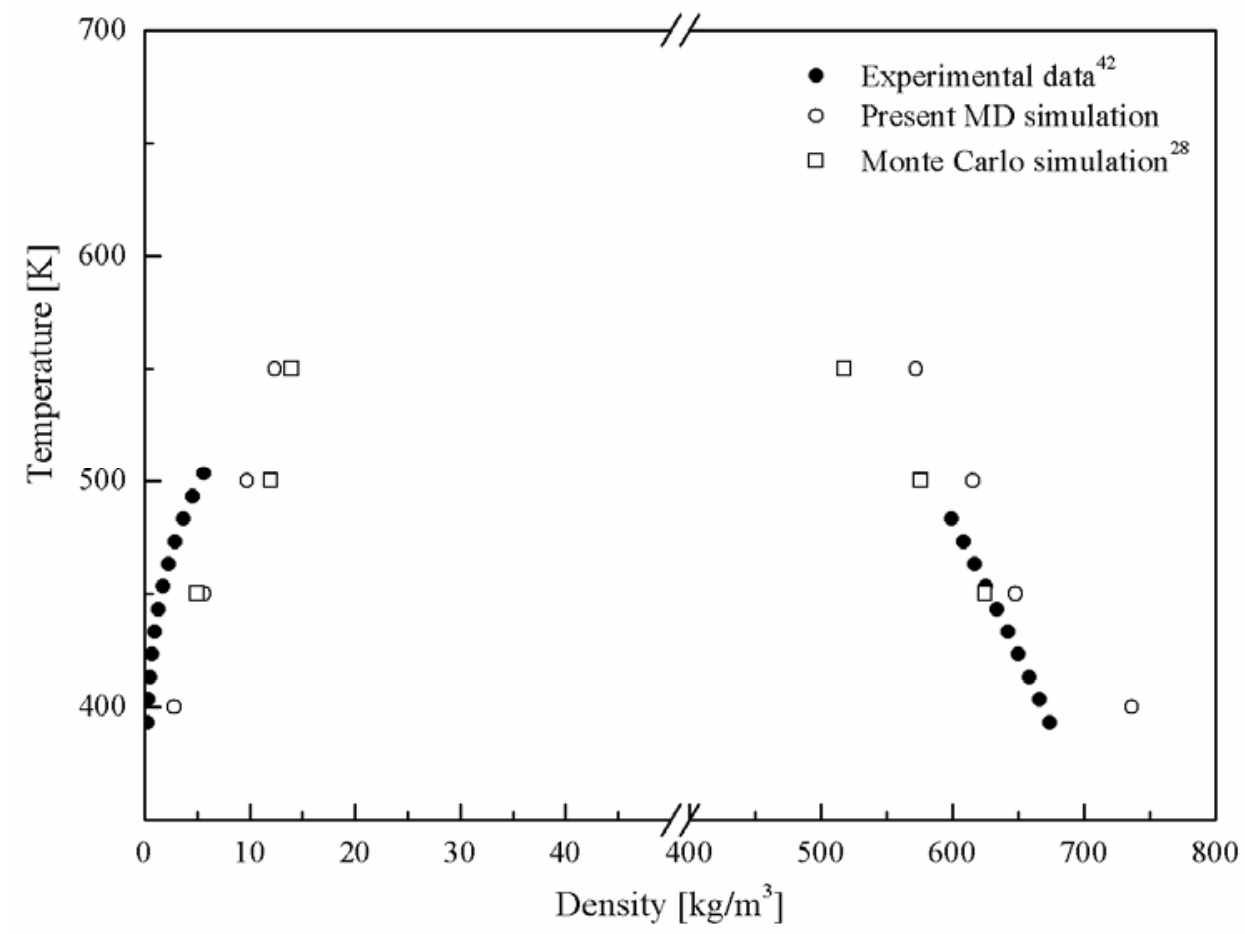

Fig. 4 


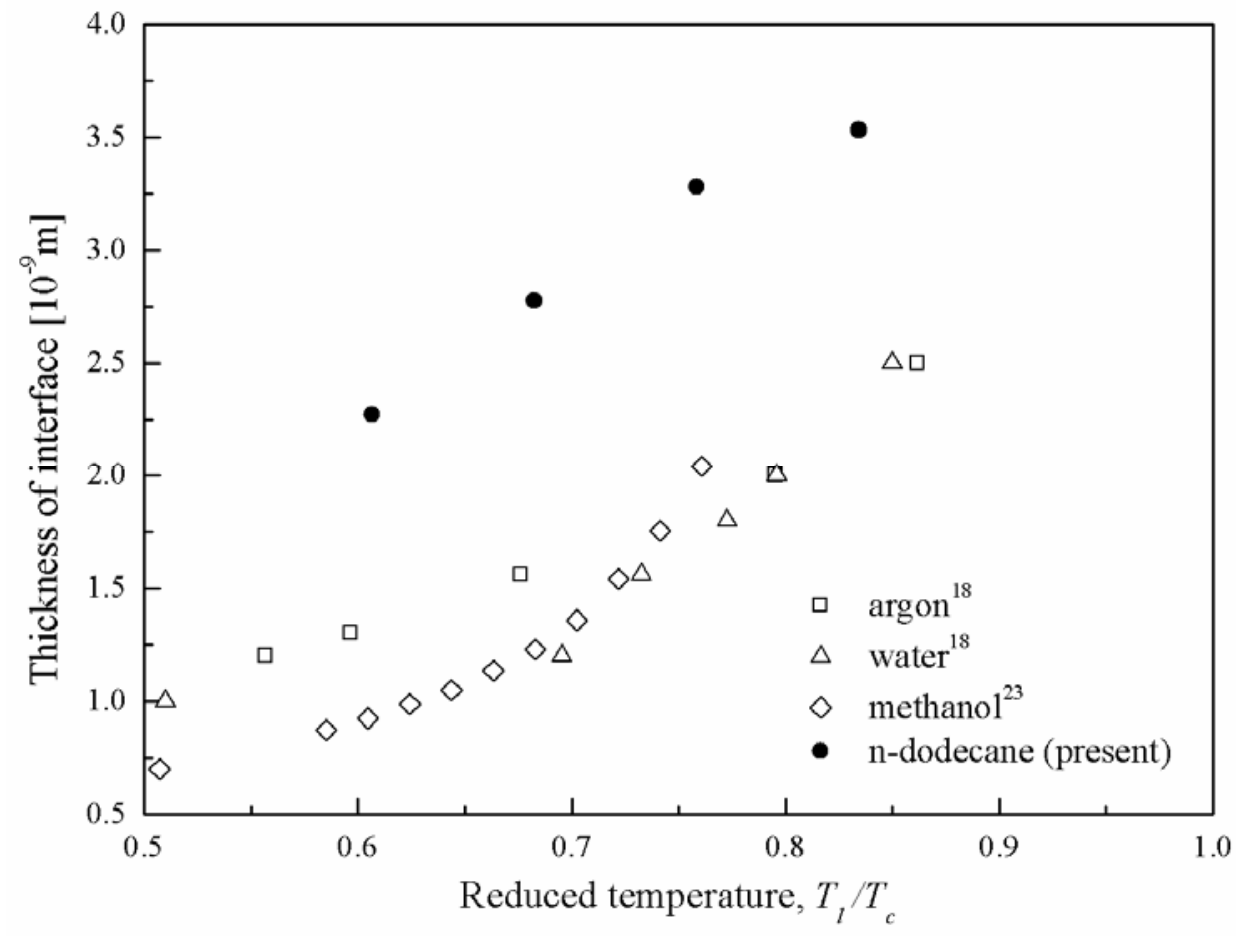

Fig. 5 

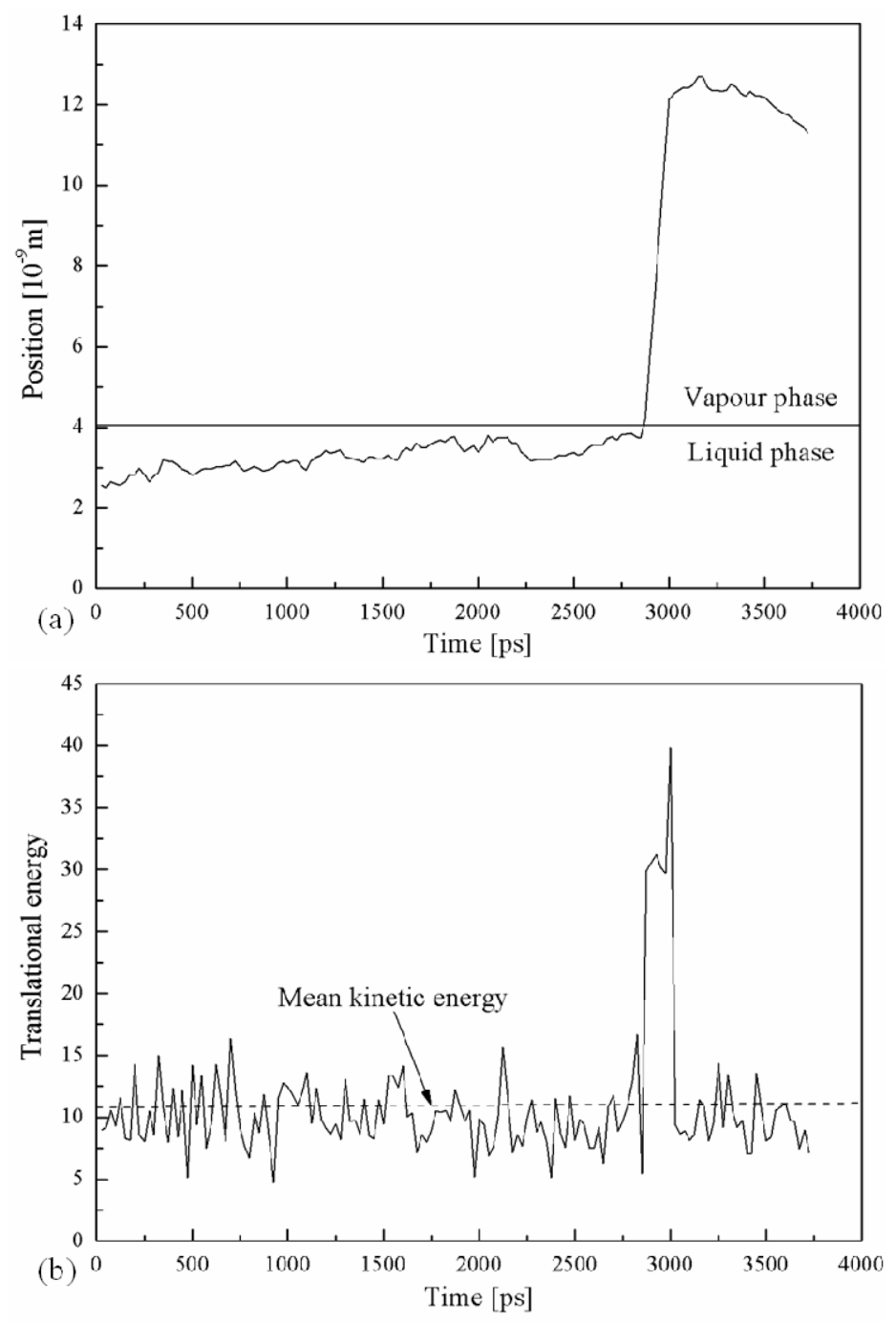

Fig. 6 

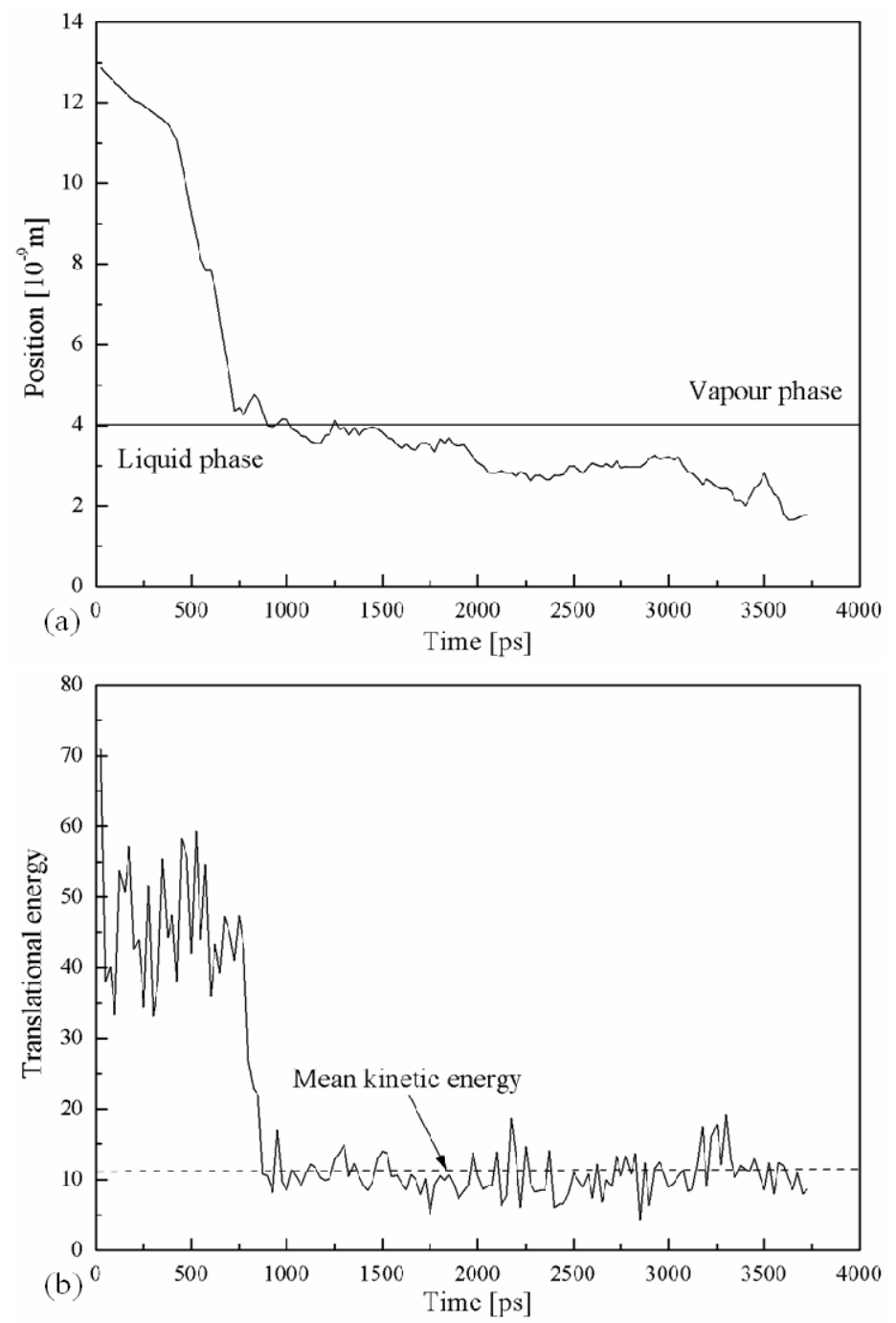

Fig. 7 

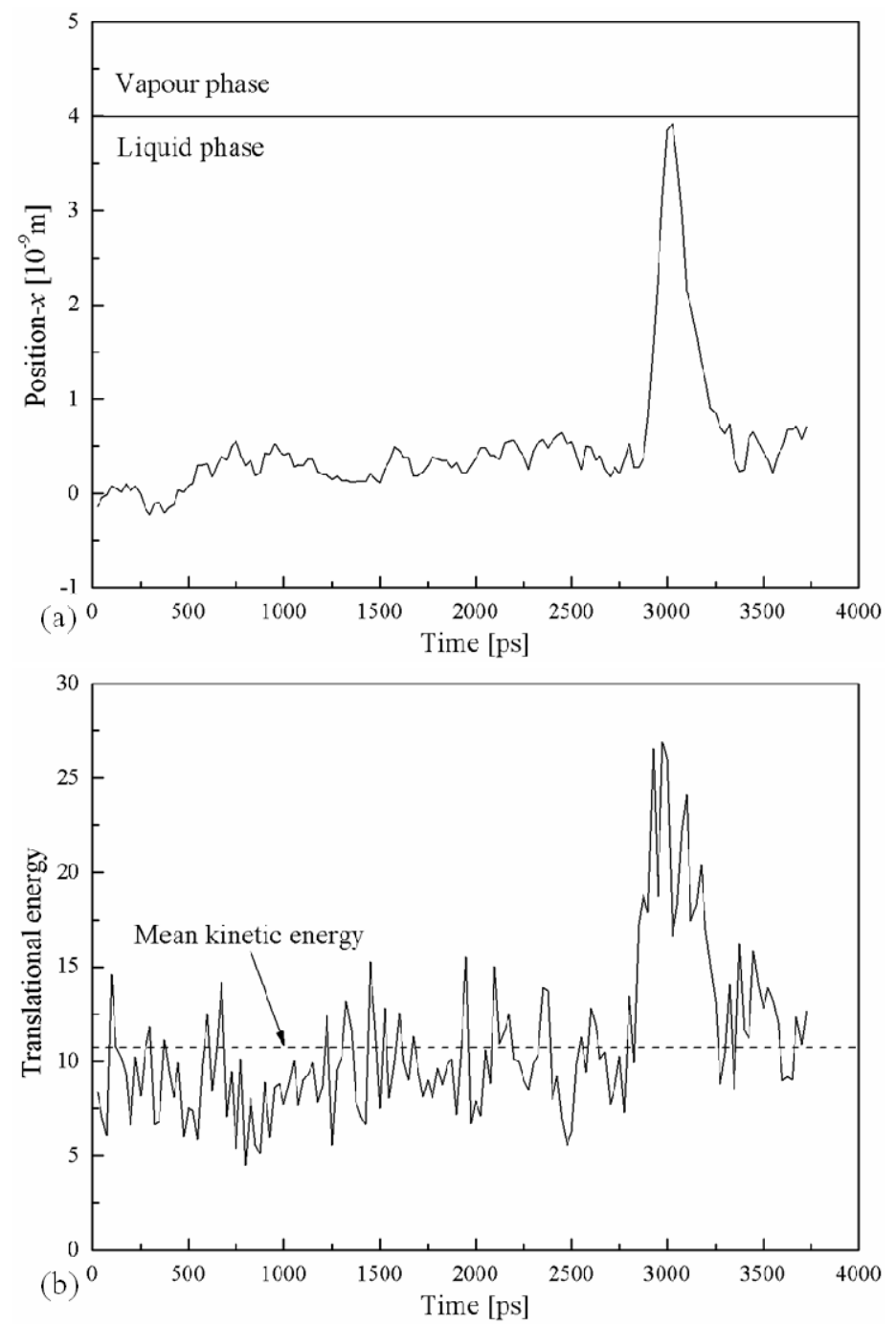

Fig. 8 

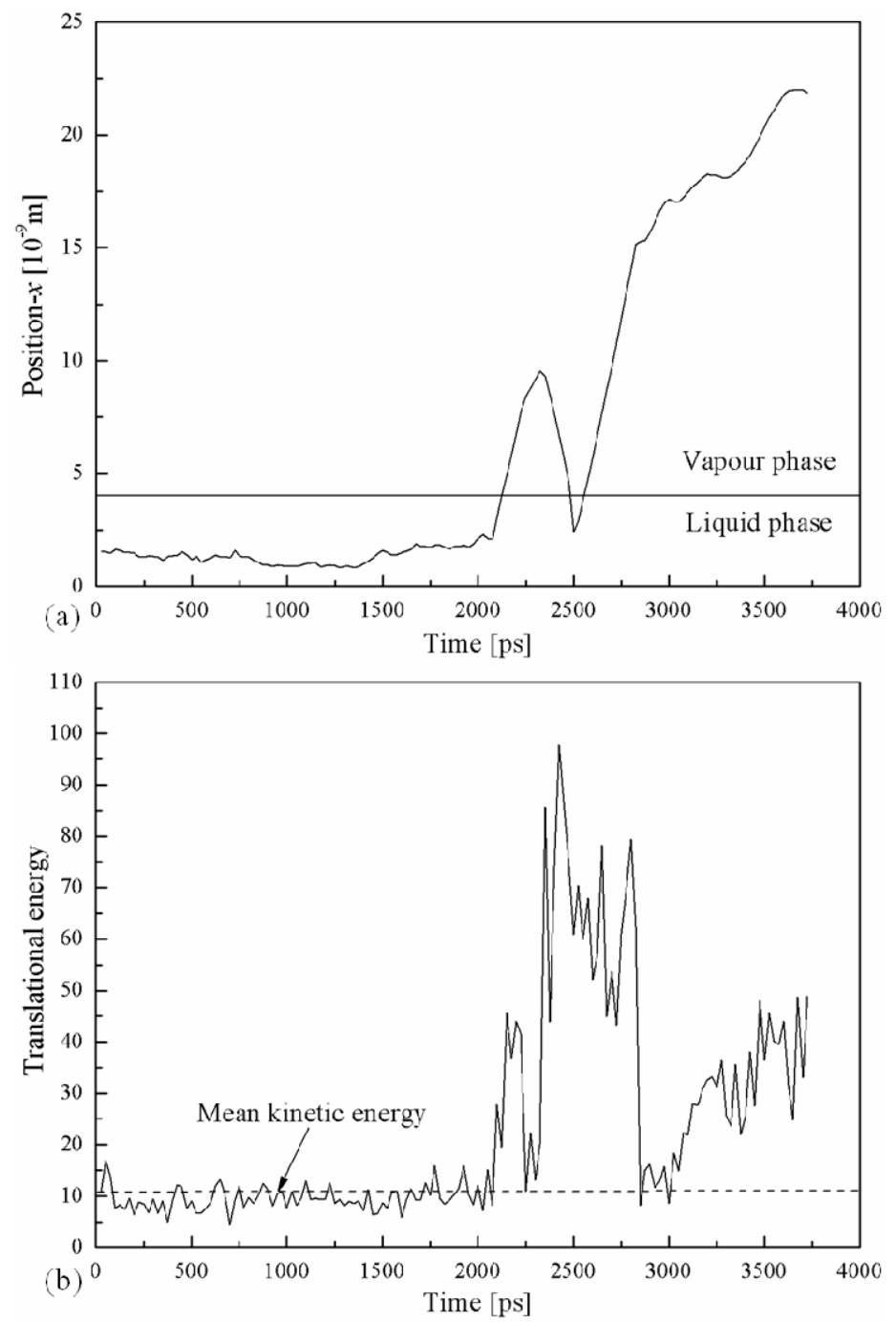

Fig. 9 


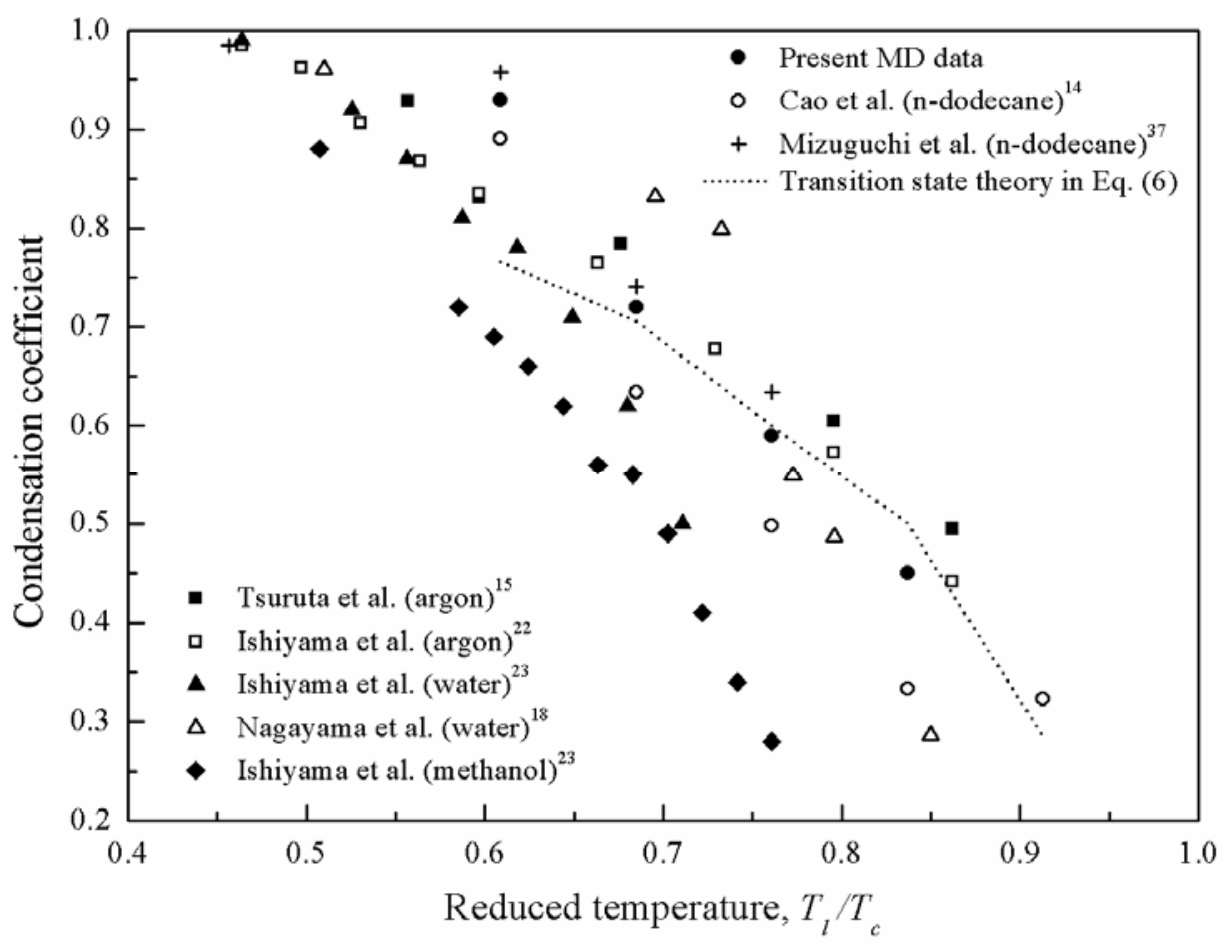

Fig. 10 

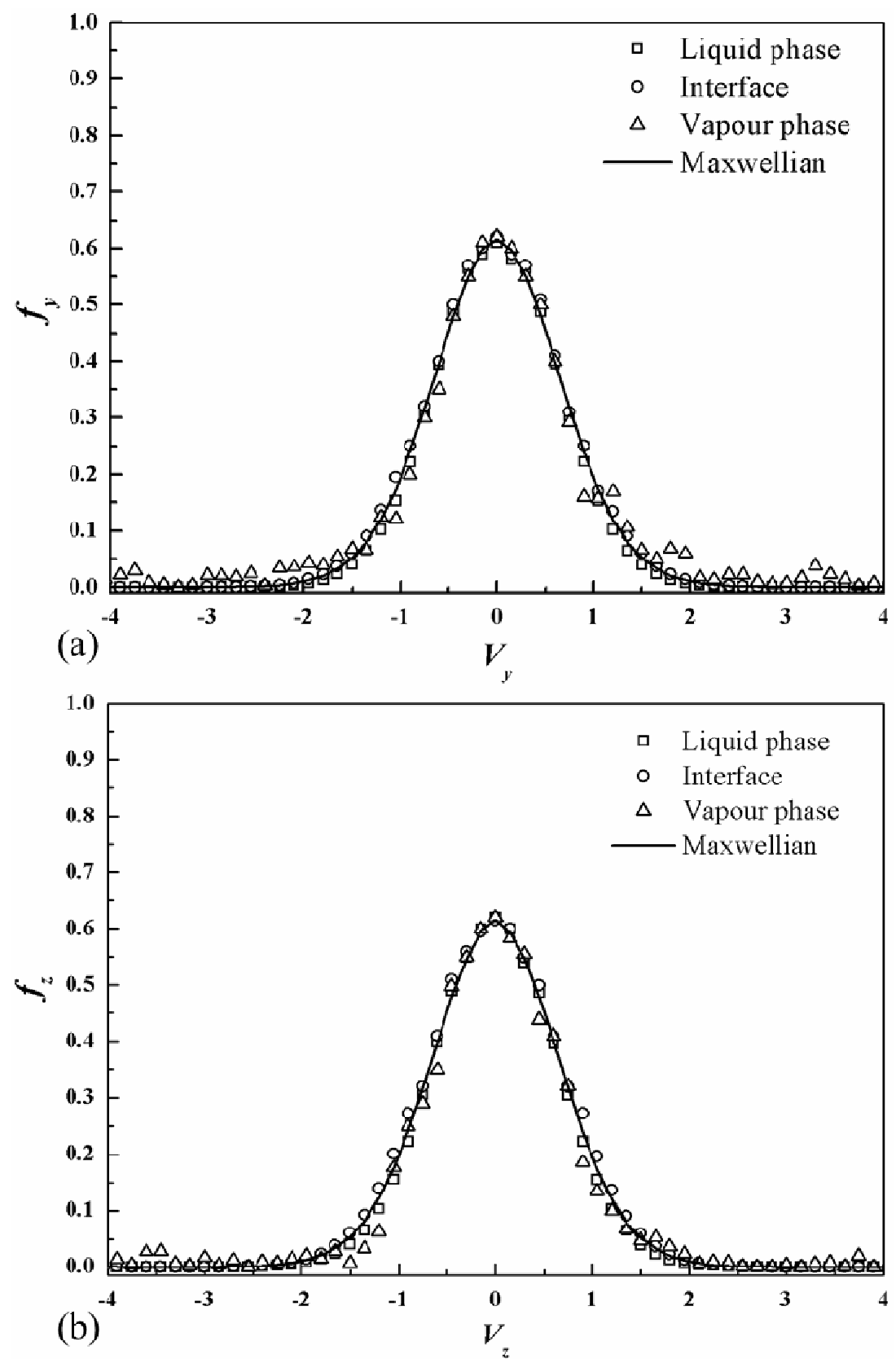

Fig. 11 

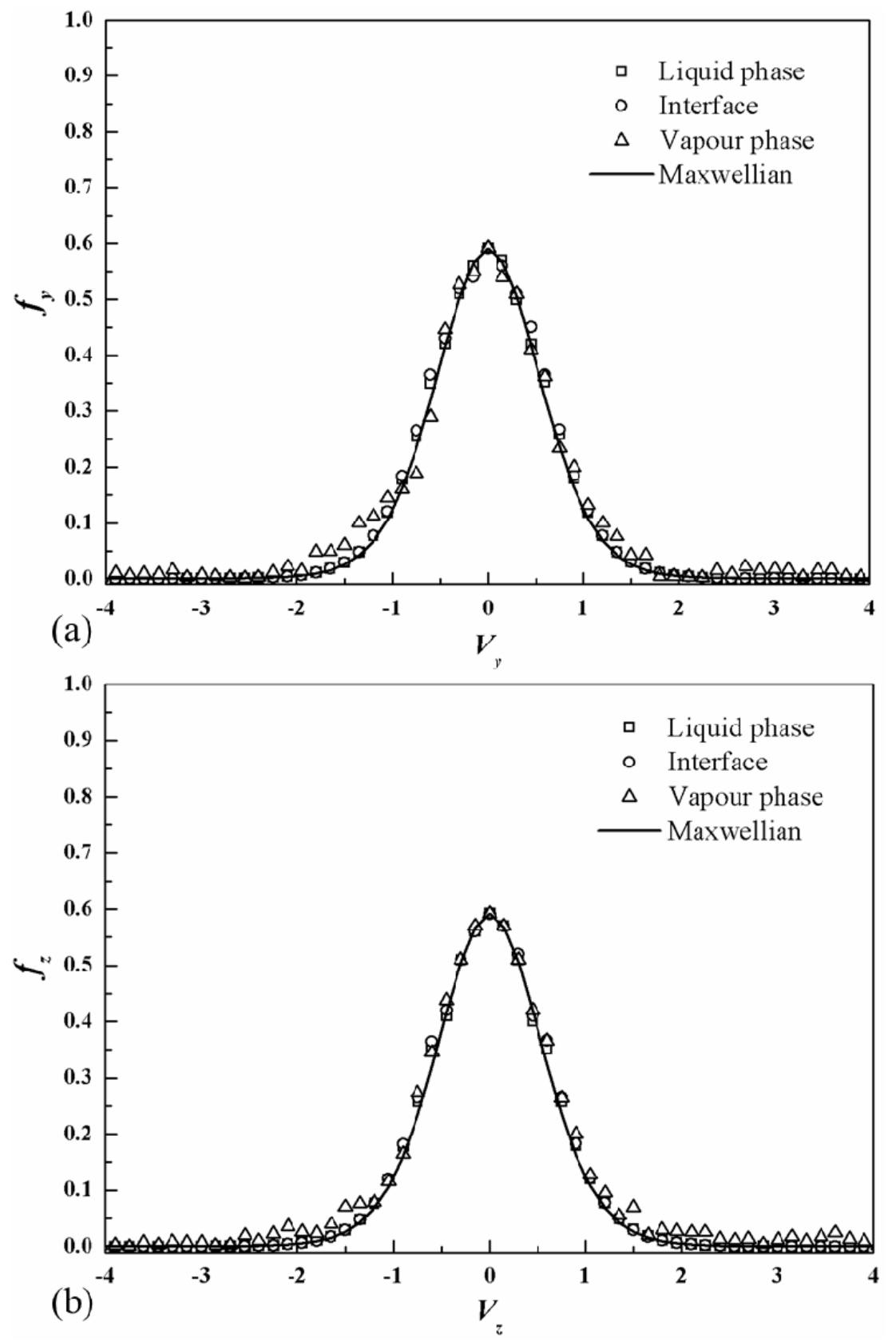

Fig. 12 


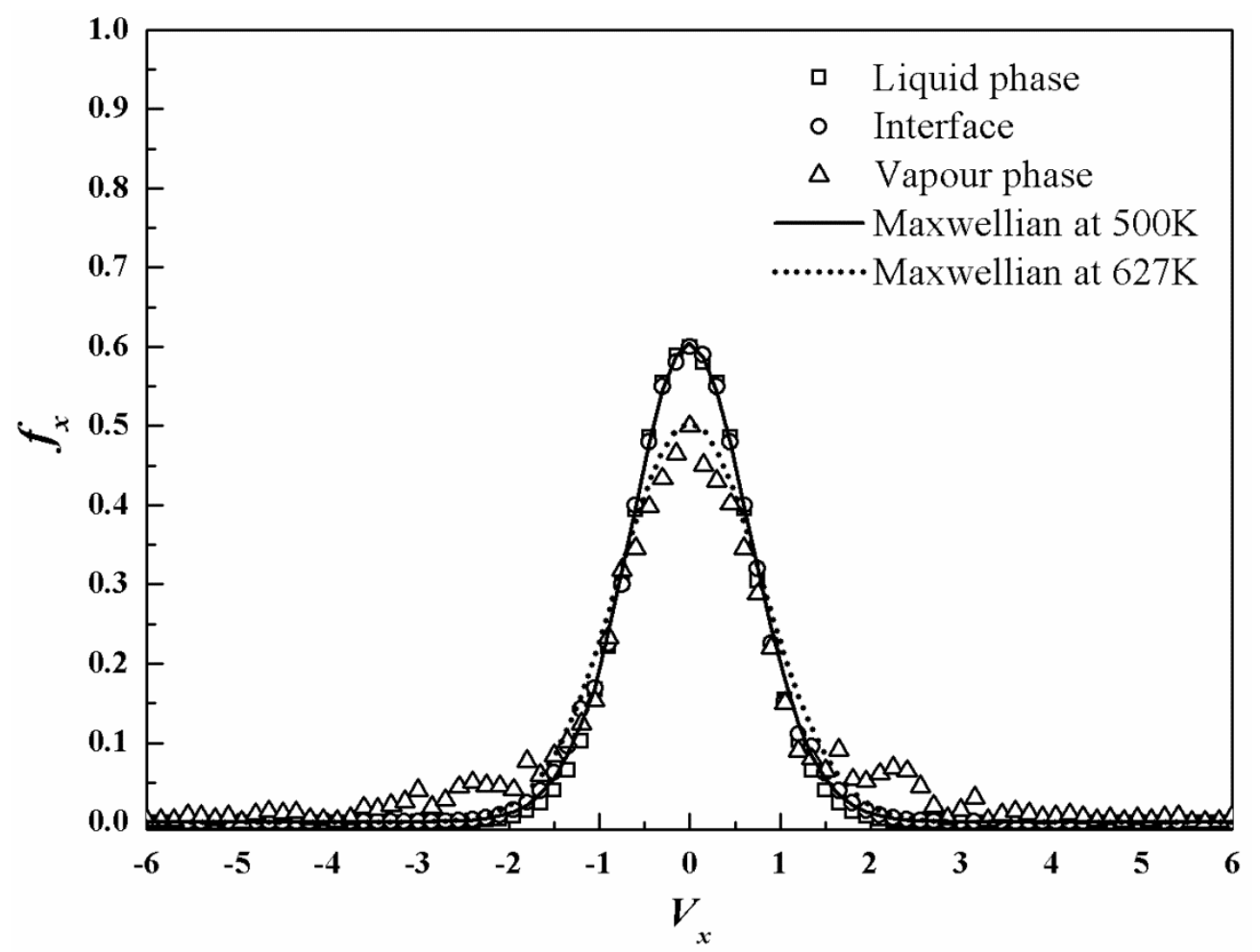

Fig. 13 


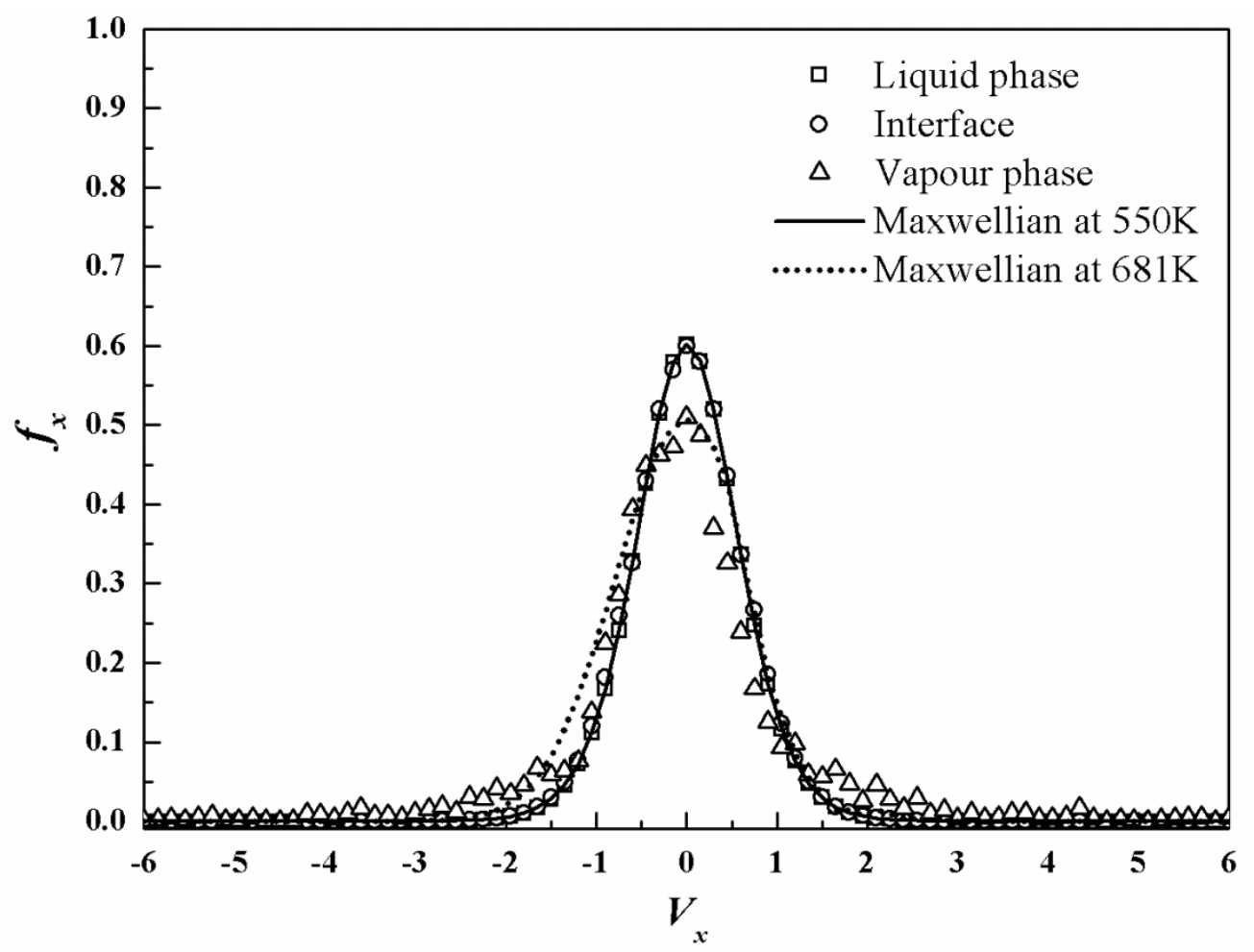

Fig. 14 